\title{
Trans-foraminal endoscopic uniportal decompression in degenerative lumbar spondylolisthesis: a technical and case report
}

Ajay Krishnan ${ }^{1,2,3^{*}}$ (D, Mahesh Kulkarni ${ }^{1}$, Mreetaunjay Singh ${ }^{1}$, Chaitanya Reddy ${ }^{1}$, Shivanand Mayi ${ }^{1}$, D. Devanand ${ }^{1}$, Ravi Ranjan Rai ${ }^{1}$ and Bharat R. Dave ${ }^{1}$

\begin{abstract}
Background: Degenerative spondylolisthesis is a common spinal pathology. Traditionally, spinal fusion is an accepted standard surgical treatment for listhesis. But fusion is a major intervention with its known pitfalls. With technological progression, minimally invasive spinal fusion (MISF) procedures are becoming mainstream. Percutaneous trans-foraminal endoscopic lumbar discectomy/decompressions (PTELD) without stabilization has many advantages over even a MISF for select group of patients.

Case presentation: In this case report, we describe using a uniportal unilateral trans-foraminal approach (TFA) for stable listhesis with lumbar disc herniation (LDH) causing chronic bilateral radicular symptoms and back pain with acute exacerbation. Under local anesthesia, we used a flat entry for PTELD, which facilitates an approach to both disc sides ventrally and even dorsal aspect lateral recess decompression on the dominant ipsilateral side. No fixation was done. An excellent outcome is obtained immediately at 6 weeks and maintained at 39 months of follow-up.

Conclusion: PTELD is worth considering as an intermediate procedure before fusion is offered in lateral recess stenosis in stable listhesis patients who have consented and understand the progressive cascade of spinal degeneration.
\end{abstract}

Keywords: Lumbar, Disc, Herniation, Stenosis, Ventral, Trans-foraminal, Percutaneous, Endoscopy

\section{Background}

Spine surgery has seen a significant progress from conventional open surgery to MISF. In today's world of percutaneous endoscopic spine surgery, PTELD and PIELD are the most minimalist options [1].

PTELD has significant advantages like a minimum surgical insult to the back muscles, less trauma, loss of blood, decreased hospital stays and speedy recovery, conservation of mobility of operated segment, and high patient satisfaction avoiding complications of fusions and general anesthesia. On the other hand,

\footnotetext{
* Correspondence: drajaykrishnan@gmail.com

${ }^{1}$ Stavya Spine Hospital \& Research Institute, Nr Nagari Hospital, Mithakhali,

Ellisbridge, Ahmedabad, Gujarat 380006, India

${ }^{2}$ BIMS Hospital, opp Sir T Hospital, near Charan Boarding, Jail Road,

Bhavnagar, Gujarat 364001, India

Full list of author information is available at the end of the article
}

contraindications of PTELD are extensive-migrated disc, spinal stenosis, spondylolisthesis, cauda equina syndrome, patients with long iliac wings, extensive disc calcification, recurrent disc herniation, and involvement of more than one level $[1,2]$. But these all contraindications are now getting challenged by pioneers and getting reported sporadically [3-5].

Here, we present a case of stable spondylolisthesis and LDH causing bilateral compression treated with PTELD with a successful outcome.

\section{Case report}

The patient came demanding for PTELD procedure. The patient was operated with an informed consent. All potential complications were explained and the nonavailability of recommendations of PTELD for stenosis/ 
listhesis was divulged. If required, the need to convert to open surgery at the time of index surgery or later was conveyed.

The patient was a 57-year-old female with a bilateral leg (left greater than right) and back pain, with marked limitation of activities of daily living for 1 year without any neurological deficit. The claudication distance was 100 steps, and standing time was 15 min since a year but got aggravated in severity for 3 weeks. She was no more able to take few steps or stand. The patient was investigated with radiograph anteroposterior and lateral static/ dynamic and MRI (Fig. 1).

With the anesthesiologist supervision, the patient was operated in a prone position under conscious sedation with local anesthesia. The side of uniportal entry was chosen on dominant radiculopathy of the left side. An imaginary line drawn to the annular puncture site of medial pedicular line in the skin and surgical trajectory is planned. This angle was $15^{\circ}$, and the skin puncture point was $14.5 \mathrm{~cm}$ from midline. A 16-gauge needle was inserted in the safe triangle and confirmed fluoroscopically with patient feedback for safety at all steps. The trajectory of the needle tip never crosses the inter-facetal plane in lateral view to avoid retroperitoneal structure till it touches the facet. A 7-mm incision was put, progressive tissue dilating single trocar and an 8-mm beveled working cannula was placed sequentially. Then, through the endoscope, an inside-out decompression was done with a removal of offending compression by nucleus pulposus fragment and collagenized hardened annulus. A Maxmore endoscope system was used (Maxmorespine ${ }^{\circ}$ by Hoogland Spine Products, Germany). A visualized endoscopic foraminoplasty and lateral recess decompression was done with endoscopic burr. Nouvag drill system was used (NOUVAG AG, Goldach, Switzerland). The amount of facet removal was progressive in millimeters at the ventral and medial part of the facet till the curtain of ligament flavum is visible. Then, the ligament flavum was cut and scrapped with articulated scoop till the left side traversing nerve root dorsal decompression could be done optimally. Adequacy of the ventral and dorsal aspect of the traversing root is ascertained by visualization, pulsation, cough impulse, and probing along its length from index disc's upper border till pedicle (Fig. 2), whereas on the opposite right side, subannular ventral decompression only is possible and was done. Opposite side dorsal recess (subarticular) decompression cannot be done by unilateral approach. Decompression confirmation was done by complete visualization of dural sac, probing, dural pulsations, irrigation flutter, and cough impulse.

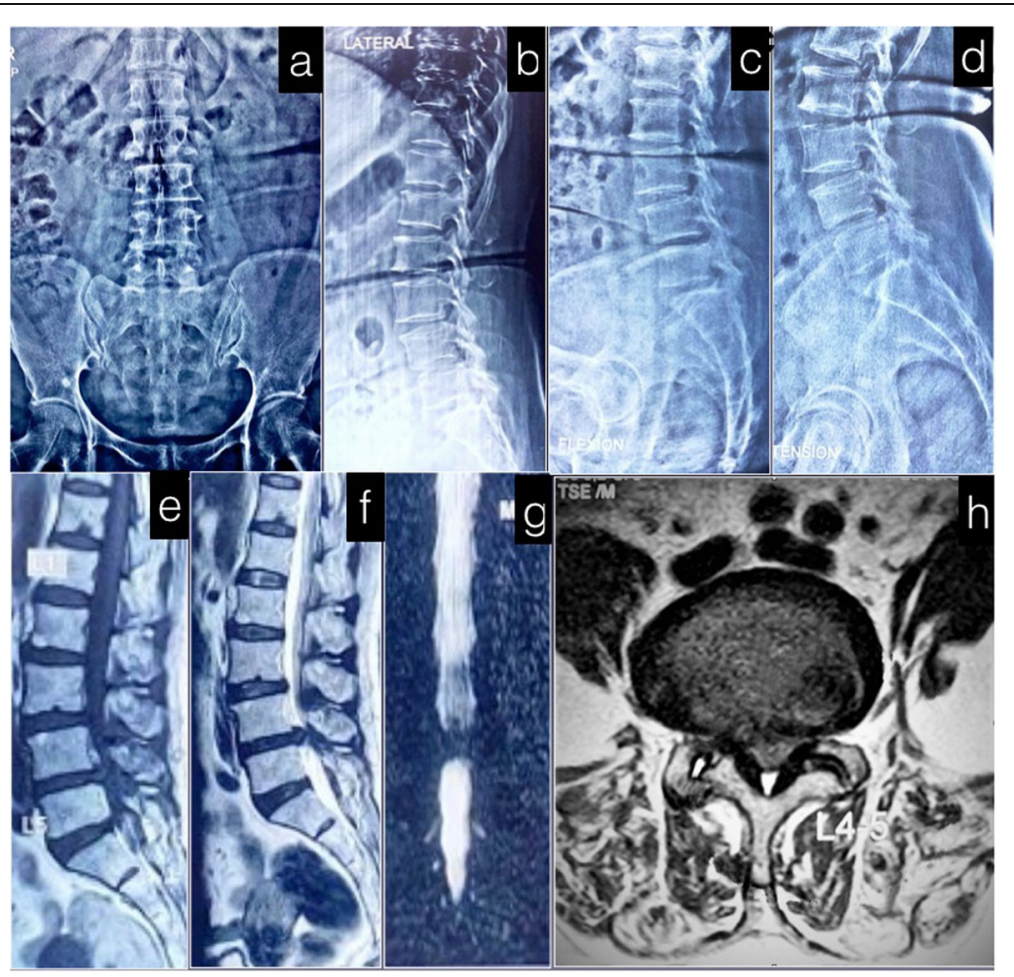

Fig. 1 a-d Antero-posterior, neutral standing lateral, flexion, and extension radiograph of the patients showing grade 1 listhesis at L4-5 level. e, $\mathbf{f}$ T1 and T2 sagittal MRI sections showing a disc prolapse with buckled annulus and reduced disc space. $\mathbf{g}$ The MR myelogram showing complete myeloblock. $\mathbf{h}$ T2 axial film showing ligamentum flavum (dorsal stenosis) with facet tropism, a buckled annulus, and disc protrusion causing ventral stenosis. Also notable is a significant sarcopenia 

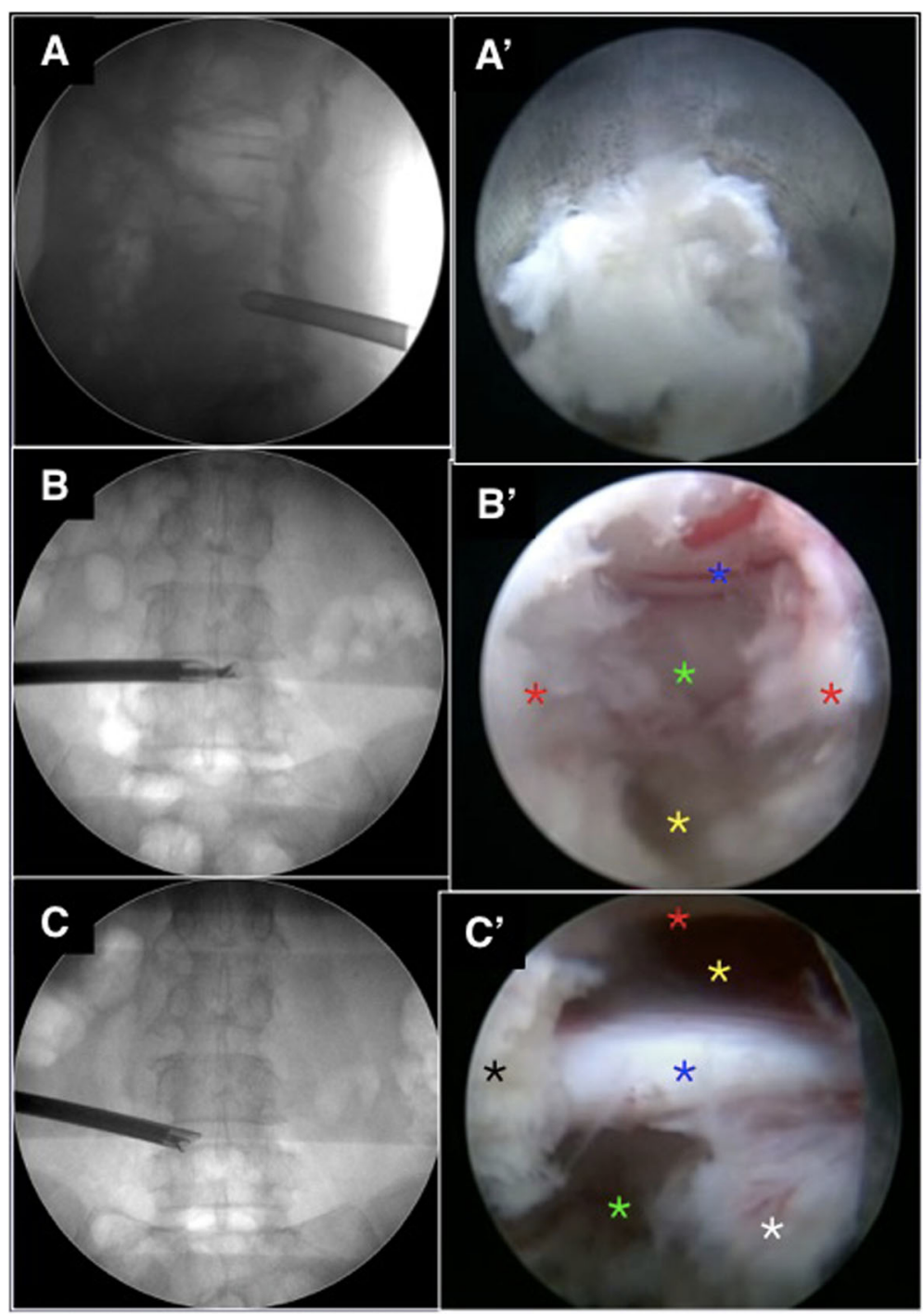

Fig. 2 A Sub-annular combo (working channel-endoscope). A' Endoscopic view of annulus. B Combo with midline crossing grasper. B' Endoscopic view, Astrix: red; annulus, green; opposite side below right traversing root, yellow; space created by removed nucleus, blue; decompressed cord. C Combo position while doing the endoscopic foraminoplasty. C' Endoscopic view, Astrix: blue; decompressed traversing root, yellow; space created dorsal to traversing root removing the LF, red; burred facet, green; ventral decompression, white; annulus and black; redundant non compressive capsule and LF* in superior foramen. * $L F$ ligamentum flavum

After retrieving the endoscope, the skin was sutured with sub-cuticular stitches. The surgery duration was $103 \mathrm{~min}$. The patient was permitted to ambulate on the same day of surgery and discharged with a firm lumbar orthosis after post-operative MRI confirmation (Fig. 3).

The evaluation of neurologic and functional outcomes was done using the validated measures of the ODI and VAS $[6,7]$. ODI improved from 95.56 to 08 at 03 weeks and maintained at 39 months of follow-up. VAS L (leg) improved from 9 left $/ 6$ right to $0 / 0$ bilateral, respectively. VAS B (back) improved from 07 to 02 . Patient satisfaction index was 03. Patient satisfaction index is a validated score with 1-3 score given for satisfaction outcome [8]. This outcome was achieved at 6 weeks and stable at 39 months of follow-up.

\section{Discussion}

Degenerative spondylolisthesis can cause back pain, radiculopathy, and clinical symptoms reflecting spinal canal stenosis [9]. For spondylolisthesis, fusion is accepted as treatment of choice because it offers surgical stabilization and decompression which is logical [9]. Scientific consensus is that the spinal fusion prevents progression of spondylolisthesis, but research shows it is not always the case [10]. There are reports of increased hypertrophic degenerative arthritis of the 


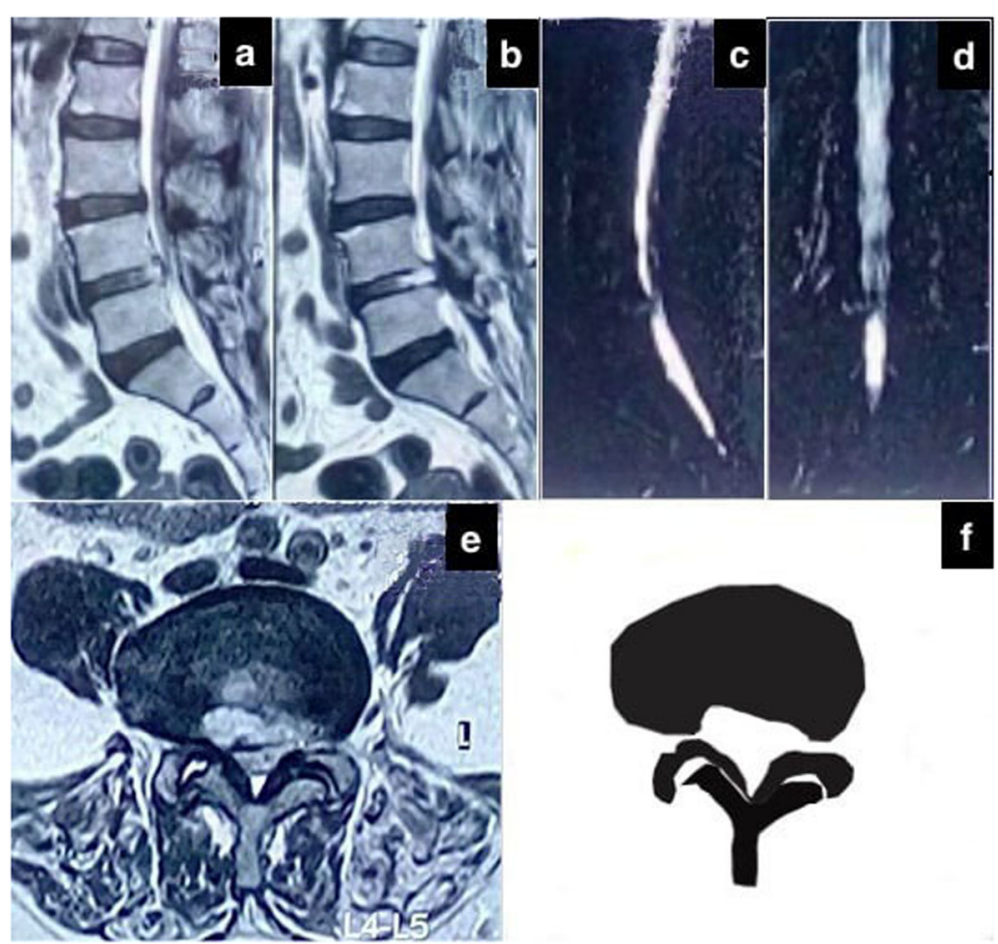

Fig. 3 Postoperative; a b T2 sagittal and para-sagittal MRI sections showing a decompressed disc prolapse (partial annulectomy and prolapsed targeted nucleus pulposus fragmentectomy). c, d MR myelogram showing myeloblock now opened up completely. e The T2 axial section showing ventral decompression starting from left side and extending to opposite right side ventral to opposite traversing root. $\mathbf{f} \|$ llustrative image of figure e showing removal of optimal disc, dorsal facet, and ligament flavum (foraminoplasty) on left side as compared to opposite right side

adjacent facet joints, spinal stenosis, severe disc degeneration, and residual pain all related to spinal fusion [11]. Even NASS have changed the terminology for recommendation in stable listhesis. In the year 2008 , the recommendation was of fusion. Now, in the year 2014 revision, the fusion is suggested if required $[12,13]$. Likely, this is due to the advances of minimal invasive non-fusion surgeries.

Conventional non-fusion decompression surgery by MLD does good decompression but with increased posterior access-related traumatization in comparison to endoscopic techniques [2]. PIELD is also a further minimal access surgery possible [1]. PTELD can achieve the ventral decompression without damaging the posterior compartment stabilizing structures [1]. PTELD can be applied in the removal of fragments via enlarged foramen which is also an appropriate approach for bilateral symptomatic disc herniation [14]. In our case, listhesis was present along LDH with bilateral compression causing radicular leg pain on both sides which could be decompressed adequately (Fig. 2).

Removal of large nucleus pulposus in case of bilateral compression can cause postoperative spinal instability and chronic pain [15]. To avoid this, we positioned the endoscope in dorsal 1/4th of the disc, removed pathological sub-annular pieces and intra-canalicular tissue, and preserved normal nucleus pulposus as much as we could (Fig. 3). We could go across to the opposite side to do the partial ventral decompression of opposite traversing root. This is possible due to the flat approach to the ventral epidural space (Fig. 4). This flat approach was first described by S. Ruetten in 2005 [16]. The flat approach is technically challenging and is fought with dangers of retro-peritoneal/abdominal injury. The planning of it may be done on pre-operative larger field of view MRI and CT) axial sections. For safety, the tip of the needle should remain dorsal to inter-facetal plane while reaching the initial target landing point and should cross it only after touching the facet complex.

Limitations do exist in PTELD. Posterior ligamentum flavum and subarticular stenosis removal is not extensively possible but optimally only addressable. Progression of stenosis can occur further in these cases. But the excellent achieved immediate and maintained outcome at 39 months justifies it as an intermediate procedure before an all-out fusion need arises. Larger series data reporting is needed for concrete recommendations before it is considered as a standard of care. Patients with mild or no back ache, with restabilization signs (osteophytic fusion, dry facetal arthrosis, gross disc space 


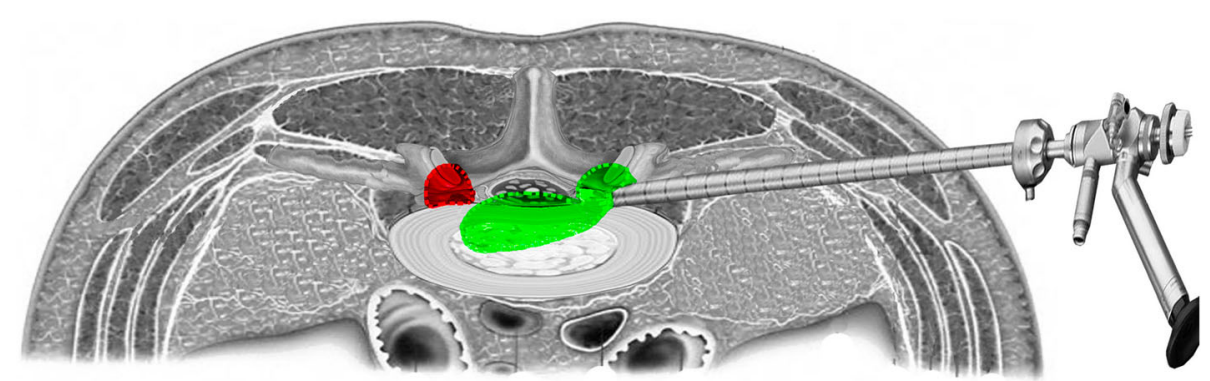

Fig. 4 Illustrative image showing the ventral reach (area green) of a flatly placed trans-foraminal endoscope. It can reach to posterior 1/3rd of the disc and up to middle of the spinal canal and across to opposite side ventral to opposite traversing root. It is best positioned to decompress ventrally (central big disc, hardened/calcified/redundant annulus, or end plate spurs without cord retraction. The endoscopic visualized foraminoplasty can decompress ipsilateral dorsal and lateral aspect of canal optimally, removing part of facet, ligamentum flavum, and capsule of facet (area green). But it cannot target the opposite subarticular stenosis (area red)

narrowing), non-major facet effusion, lordotic disc angle on dynamic radiographs, and less than $3 \mathrm{~mm}$ translation are all signs of a stable listhesis and can be saved from fusion. But the potentially unstable or unstable listhesis are the one that needs fusion [17]. This is now well understood but still less followed. The patients of degenerative listhesis who wants some improvement without going through a surgery that requires general anesthesia, an inpatient hospital stay, and the possible morbidities associated with an instrumented fusion procedure may be offered PTELD as an intermediate procedure [4]. The surgery by TFA can decompress the ventral stenosis unilaterally or bilaterally and the ipsilateral subarticular dorsal stenosis as well [4, 5, 18]. But it cannot address the subarticular opposite side stenosis. A bilateral approach has also been mentioned for endoscopic decompression addressing bilaterally symptomatic listhesis with addition of percutaneous fusion [19].

The recent most literature has come out with a cases series ( $n=21, n=26, n=4)$ with good to excellent outcomes with removed ventral bone to achieve lateral recess decompression adequately $[4,5,18]$. Li et al.'s focus was in removing of the posterosuperior margin of the inferior vertebral body in 26 cases of low-grade listhesis (Fig. 5) [5]. Their patient group had dominant leg symptoms and no back pain, and the symptoms were unilateral leg pain. The outcome of 21 patient of Jasper et al. was also encouraging but with shorter follow-up of 1 year only. They also removed all the three components of the stenosis that is ventral articular process, disc herniation, and part of the superior endplate [4]. Their symptoms were also unilateral leg

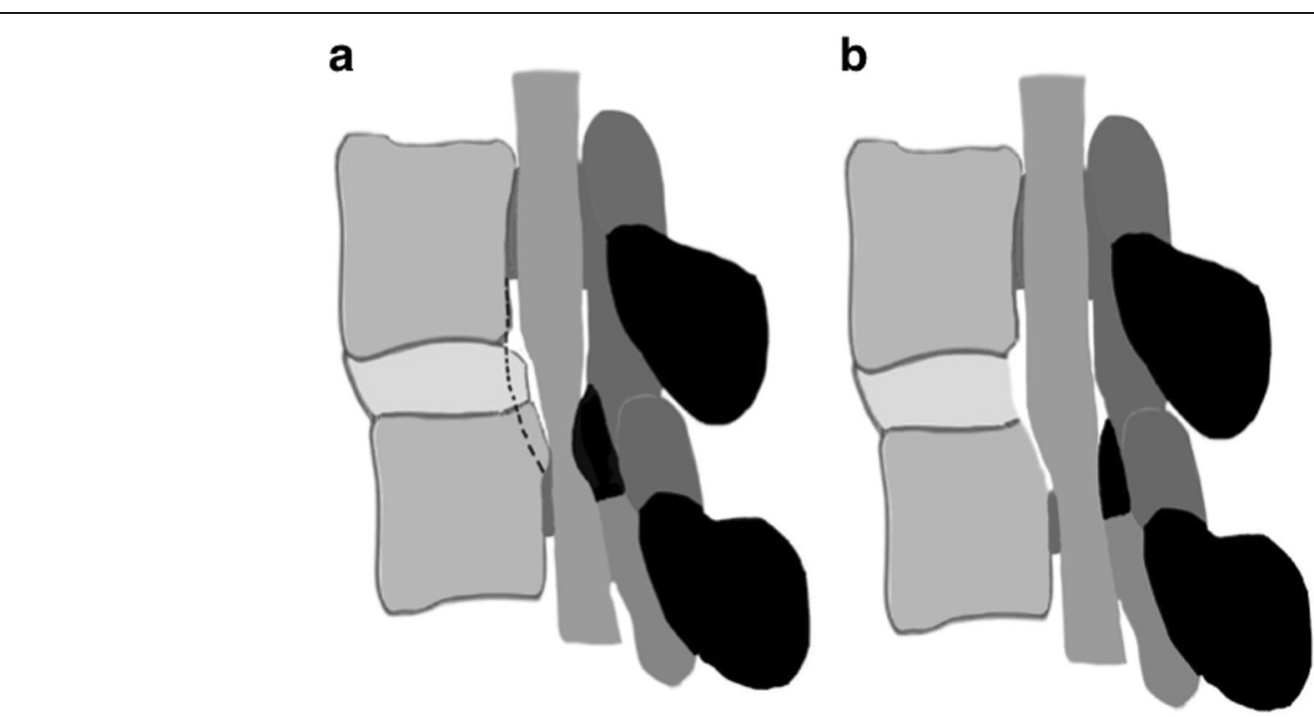

Fig. 5 a, b Illustration showing Li et al.'s [5] approach. The lateral recess has a ventral and dorsal component. A good ventral decompression and a marginal dorsal decompression by foraminoplasty can give adequate space for the trapped nerves 


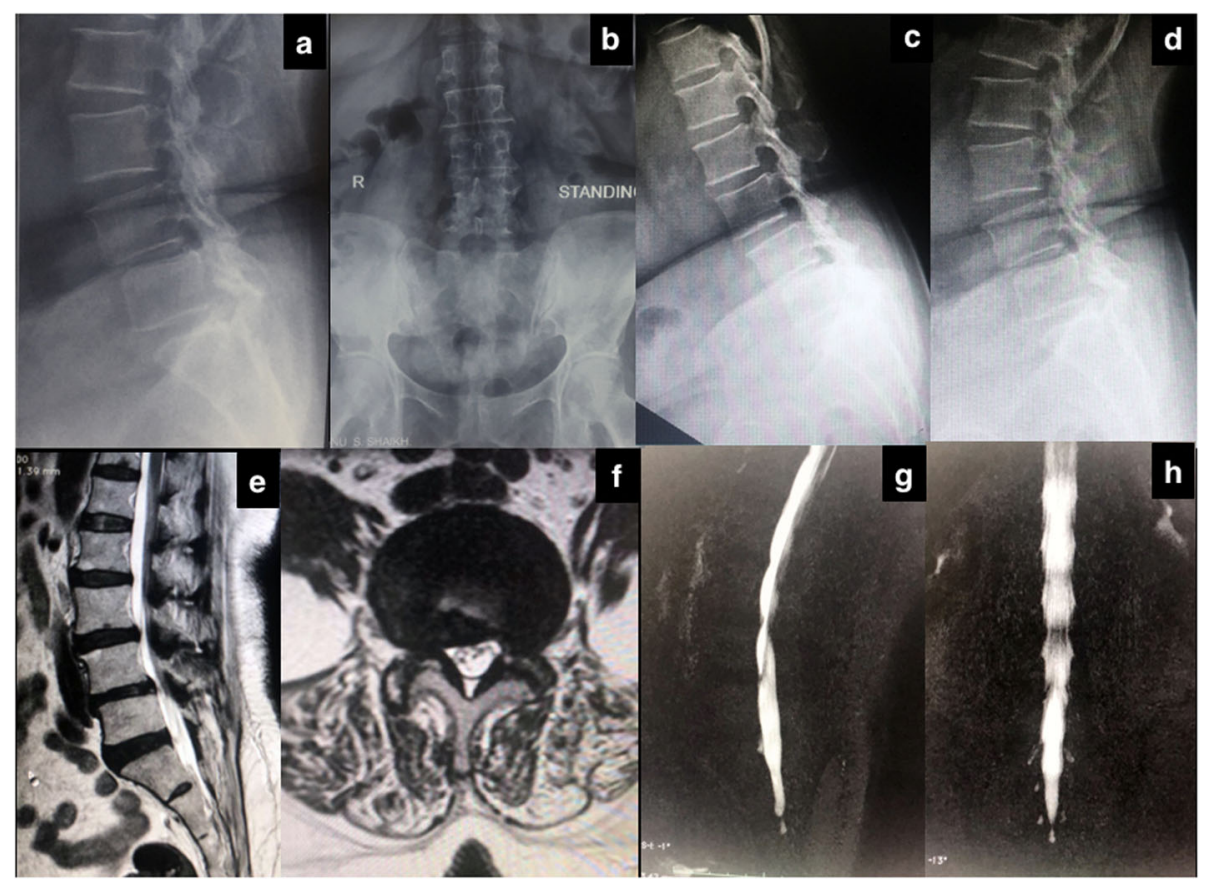

Fig. $\mathbf{6}$ a-d At follow-up standing antero-posterior, neutral standing lateral, flexion, and extension radiograph of the patients showing stable listhesis. e, f T2 axial and para-saggital MRI sections showing maintained decompression. $\mathbf{g}, \mathbf{h}$ The MR myelogram showing no compression

pain without back pain. Telfien et al.'s four cases were in the degenerative lumbar scoliosis with lateral subluxation, where disc prolapse was the main component and it was considered an elegant salvage approach for elderly (average age 74.8 years) [18]. In our index case, the patient had significant back pain and bilateral symptoms all of which got relieved significantly and maintained at final follow-up (Fig. 6). Some literatures are now questioning the potentiality of instability in listhesis. Facet effusion also is not always a sign of instability [20]. Our patient had facet tropism, sagittal facets, and effusion (Fig. 1h), but was still stable. Moreover, the patient also had significant sarcopenia. Literature reports of sarcopenia compounding the failures in lumbar surgery are also getting reported [21]. An open fusion or MISF would have further devitalized the muscles and added new problems which were completely avoided by doing a PTELD under local anesthesia.

So, the science is evolving and alternative nonconventional techniques will also find favors if applied safely in some patients. PTELD offers noticeable advantages and should be considered as an intermediate procedure for selected demanding patients where surgeon has extensive experience with open and endoscopic approaches.

\section{Conclusion}

PTELD is an effective technique to do ventral decompression with the disc as the main component of stenosis in a case of stable listhesis with predominant unilateral symptoms. The unilateral approach cannot decompress the opposite side dorsal stenosis. It is not a permanent replacement for fusion. PTELD should only be considered as an intermediate procedure for selected demanding patients.

\section{Abbreviations}

CT: Computed tomography; LDH: Lumbar disc herniation; MISF: Minimally invasive spinal fusion; MLD: Microscope-assisted lumbar decompression; MRI: Magnetic resonance imaging; NASS: North American Spine Society; ODI: Oswestry Disability Index; PIELD: Percutaneous inter-laminar approach; PTELD: Percutaneous trans-foraminal endoscopic lumbar discectomy/decompressions; TFA: Trans-foraminal approach; VAS: Visual analog score

\section{Acknowledgements}

None

\section{Authors' contributions}

AK contributed as the Chief operating surgeon and primary manuscript writer and director. CR contributed to the image processing and computer work. MK and MS contributed to the referencing work. SM and DD contributed to the editing and proofreading. BRD is the Director of the Institute. All authors read and approved the final manuscript.

\section{Funding}

Not applicable.

Availability of data and materials Not applicable.

Ethics approval and consent to participate Not applicable. 


\section{Consent for publication}

Written informed consent for the publication of the personal details and images that appear in this manuscript has from the patient.

\section{Competing interests}

The authors declare that they have no competing interests.

\section{Author details}

'Stavya Spine Hospital \& Research Institute, Nr Nagari Hospital, Mithakhali, Ellisbridge, Ahmedabad, Gujarat 380006, India. ${ }^{2}$ BIMS Hospital, opp Sir T Hospital, near Charan Boarding, Jail Road, Bhavnagar, Gujarat 364001, India. ${ }^{3}$ Ahmedabad, India.

Received: 7 June 2019 Accepted: 16 October 2019

Published online: 31 October 2019

\section{References}

1. Choi G, Pophale CS, Patel B, Uniyal P. Endoscopic spine surgery. J Korean Neurosurg Soc. 2017;60(5):485-97.

2. Ruetten S, Komp M, Merk H, Godolias G. Full-endoscopic interlaminar and transforaminal lumbar discectomy versus conventional microsurgical technique: a prospective, randomized, controlled study. Spine. 2008; 33(9):931-9.

3. Ren C, Li Y, Qin R, Sun P, Wang P. Transforaminal endoscopic lumbar discectomy for lumbar disc herniation causing bilateral symptoms. World Neurosurg. 2017;106:413-21.

4. Jasper GP, Francisco GM, Telfeian AE. Transforaminal endoscopic discectomy with foraminoplasty for the treatment of spondylolisthesis. Pain Physician. 2014;17(6):E703-8.

5. $\quad L i X F$, Jin $L Y, L v Z D$, Su XJ, Wang $K$, Song $X X$, et al. Endoscopic ventral decompression for spinal stenosis with degenerative spondylolisthesis by partially removing posterosuperior margin underneath the slipping vertebral body: technical note and outcome evaluation. World Neurosurg. 2019:126:e517-25.

6. Fairbank JC, Pynsent PB. The Oswestry disability index. Spine. 2000;25(22): 2940-53.

7. Zanoli G, Strömqvist B, Jönsson B. Visual analog scales for interpretation of back and leg pain intensity in patients operated for degenerative lumbar spine disorders. Spine. 2001;26(21):2375-80.

8. Toyone T, Tanaka T, Kato D, Kaneyama R, Otsuka M. Patients' expectations and satisfaction in lumbar spine surgery. Spine. 2005;30(23):2689-94.

9. Kleinstueck FS, Fekete TF, Mannion AF, Grob D, Porchet F, Mutter U, Jeszenszky D. To fuse or not to fuse in lumbar degenerative spondylolisthesis: do baseline symptoms help provide the answer? Eur Spine J. 2012;21(2):268-75.

10. Fredrickson BE, Baker D, McHolich WJ, Yuan HA, Lubicky JP. The natural history of spondylosis and spondylolisthesis. J Bone Joint Surg Am. 1984; 66(5):699-707.

11. Lee CK. Accelerated degeneration of the segment adjacent to a lumbar fusion. Spine. 1988;13(3):375-7.

12. Matz PG, Meagher RJ, Lamer T, Tontz WL Jr, Annaswamy TM, Cassidy RC, et al. Guideline summary review: an evidence-based clinical guideline for the diagnosis and treatment of degenerative lumbar spondylolisthesis. Spine J. 2016:16(3):439-48.

13. Watters WC III, Bono CM, Gilbert TJ, Kreiner DS, Mazanec DJ, Shaffer WO, et al. An evidence-based clinical guideline for the diagnosis and treatment of degenerative lumbar spondylolisthesis. Spine J. 2009;9(7):609-14.

14. Xin G, Shi-Sheng H, Hai-Long Z. Morphometric analysis of the YESS and TESSYS techniques of percutaneous transforaminal endoscopic lumbar discectomy. Clin Anat. 2013;26(6):728-34.

15. Lee SH, Choi KC, Baek OK, Kim HJ, Yoo SH. Percutaneous endoscopic intra-annular subligamentous herniotomy for large central disc herniation: a technical case report. Spine. 2014;39(7):E473-9.

16. Ruetten S, Komp M, Godolias G. An extreme lateral access for the surgery of lumbar disc herniations inside the spinal canal using the full-endoscopic uniportal transforaminal approach-technique and prospective results of 463 patients. Spine. 2005;30(22):2570-8.

17. Simmonds AM, Rampersaud YR, Dvorak MF, Dea N, Melnyk AD, Fisher CG. Defining the inherent stability of degenerative spondylolisthesis: a systematic review. J Neurosurg Spine. 2015;23(2):178-89.
18. Telfeian AE, Oyelese A, Fridley J, Gokaslan ZL. Transforaminal endoscopic decompression in the setting of lateral lumbar spondylolisthesis. World Neurosurg. 2018;117:321-5

19. Osman SG. Endoscopic transforaminal decompression, interbody fusion, and percutaneous pedicle screw implantation of the lumbar spine: a case series report. Int J Spine Surg. 2012;6:157-66.

20. Tamai K, Kato M, Konishi S, Matsumura A, Hayashi K, Nakamura H. Facet effusion without radiographic instability has no effect on the outcome of minimally invasive decompression surgery. Global Spine J. 2017;7(1):21-7.

21. Inose H, Yamada T, Hirai T, Yoshii T, Abe Y, Okawa A. The impact of sarcopenia on the results of lumbar spinal surgery. Osteoporos Sarcopenia. 2018;4(1):33-6.

\section{Publisher's Note}

Springer Nature remains neutral with regard to jurisdictional claims in published maps and institutional affiliations.

\section{Submit your manuscript to a SpringerOpen ${ }^{\circ}$ journal and benefit from:}

- Convenient online submission

- Rigorous peer review

- Open access: articles freely available online

- High visibility within the field

- Retaining the copyright to your article

Submit your next manuscript at $\boldsymbol{\nabla}$ springeropen.com 